\title{
COVID-19, child's behavior problems, and mother's anxiety and mentalization: A mediated moderation model
}

\author{
Daphna G. Dollberg ${ }^{1} \cdot$ Keren Hanetz-Gamliel ${ }^{1}$. Sigal Levy ${ }^{2}$
}

Accepted: 2 November 2021

(c) The Author(s), under exclusive licence to Springer Science+Business Media, LLC, part of Springer Nature 2021

\begin{abstract}
We examined the direct and indirect links between COVID-19, maternal anxiety symptoms, and child behavior problems as well as the mediation-moderation links of mothers' anxiety symptoms and mentalization skills with the prediction of child behavior problems. A sample of 140 Israeli mothers with preschool children comprised the study's two groups: A COVID19 group $(n=53)$, recruited shortly after the pandemic outbreak, and a pre-COVID-19 group ( $\mathrm{n}=87)$, recruited prior to the pandemic. Mothers completed online questionnaires regarding their own anxiety symptoms (BSI anxiety subscale) and their children's internalizing and externalizing behaviors (CBCL). Maternal mentalization was assessed via the mind-mindedness representational procedure. Findings showed that mothers in the COVID-19 group experienced more anxiety symptoms and perceived their children as having more externalizing and internalizing behaviors compared to mothers in the pre-COVID-19 group. Mothers' anxiety symptoms mediated the effect of the pandemic on children's behaviors so that the higher the mothers' anxiety, the more externalizing and internalizing behaviors among the children. Mothers' mentalization moderated the association between the pandemic and the children's externalizing behaviors. Specifically, when mothers showed higher mentalization skills (higher mind-mindedness), the indirect effect of anxiety on the link between COVID-19 and children's externalizing behaviors was weaker compared to when mothers showed lower mentalization skills (lower mind-mindedness). The implications of these findings for preventive and treatment interventions that aim to reduce maternal anxiety and enhance mentalization skills to prevent children's behavior problems in the context of COVID-19 are discussed.
\end{abstract}

Keywords COVID-19 - Maternal mentalization · Mother's anxiety $\cdot$ Child's behavior problems $\cdot$ Child externalizing and externalizing behaviors

The outbreak of the SARS-CoV-2 novel coronavirus (COVID-19) pandemic has led to a significant increase in mental health problems in many countries around the world (e.g., Gavin et al., 2020; Holmes et al., 2020; Torales et al., 2020), particularly anxiety (Luo et al., 2020; Malhotra et al., 2020; Mazza et al., 2020). The social distancing regulations and lockdowns that have been enforced in many countries as a means of containing the virus's spread may be responsible for much of this anxiety (Brooks et al., 2020; Imran et al., 2020; Lei et al., 2020). Israel, like most countries around

Daphna G. Dollberg

daphna@mta.ac.il

1 School of Behavioral Sciences, Academic College of Tel Aviv-Yaffo, Tel Aviv-Yaffo, Israel

2 Statistics Education Unit, Academic College of Tel Aviv-Yaffo, Rabenu Yeruham St, P.O.B. 8401, 6818211 Yaffo, Israel the world, has experienced devastating health, economic, and social effects of the pandemic. A large national survey conducted by Israel's Central Bureau of Statistics (CBS) in May 2020 (CBS, 2020) found that $22.5 \%$ of surveyed adults (aged 21 and above) reported an increase in mental health difficulties, with anxiety and stress being the most prominent symptoms, experienced by $34.3 \%$ of the responders.

COVID-19 is also associated with increased risks for emotional and behavioral problems in children (DomínguezÁlvarez et al., 2020; Ghosh et al., 2020). Reports from around the world have documented an increase in children's fears, inattention, irritability, regression, and clinginess (Jiao et al., 2020), as well as difficulties in concentration, restlessness, nervousness, and worries (Orgilés et al., 2020). It has also been noted that preschoolers are showing more vulnerability and behavioral problems compared to older children (Romero et al., 2020). Home confinement appears to have increased the risk of children developing behavior problems 
(Romero et al., 2020). Similarly, in the Israeli survey cited above (CBS, 2020), 25.8\% of the responders said that their children's emotional state had worsened since the COVID19 outbreak. The global rise in children's COVID-19-related behavioral and emotional difficulties has been attributed to the unique combination of the health crisis, the related economic recession, and disruption of families' daily routines, home confinement, social isolation, and physical distancing from extended family, classmates, and teachers (Golberstein et al., 2020; Imran et al., 2020).

In light of these reports, the first goal of the current study was to assess differences in levels of children's problematic behaviors and their mothers' anxiety symptoms in the context of the COVID-19 outbreak vs. the pre-COVID era, using samples that had similar demographics. Furthermore, we were interested in examining to what degree the pandemic impacted children's behavior problems directly, and to what degree the impact was indirect, mediated via their mothers' anxiety and mentalizing abilities.

\section{Maternal Anxiety, COVID-19, and Child's Behavior Problems}

As noted above, COVID-19 has been associated with an increase in anxiety, particularly among mothers. Maternal anxiety is considered a risk factor for children's development, predicting later emotional, cognitive, and behavioral difficulties (Behrendt et al., 2019) in clinical and non-clinical samples (Gibler et al., 2018). Studies conducted by the current researchers in Israel prior to the pandemic showed that parents' anxiety symptoms were linked with reports of their children's externalizing and internalizing behaviors (Hanetz-Gamliel et al., 2018; Dollberg et al., 2021). Moreover, it is well established that in the presence of external threats, primary caregivers' emotional reactions, particularly their anxiety levels, play a significant role in their offspring's responses (Cobham et al., 2016; Masten \& Narayan, 2012). Thus, not surprisingly, recent research found that parents' depression and anxiety symptoms during the pandemic were associated with increases in children's behavior problems and particularly internalizing behaviors (Saddik et al., 2020; Whittle et al., 2020).

The rise in maternal anxiety in the wake of the COVID19 outbreak may stem from the medical, economic, and social threats associated with the outbreak as well as from the parental stress that has been linked with it. The virus and the measures taken to control its spread, (i.e., home confinement, school closures, and social distancing) have resulted in parents juggling between working from home and assisting their children with their remote homeschooling tasks, all of which has reportedly resulted in high parental stress and likely increased parents' mental health difficulties
(Coyne et al., 2020). Furthermore, parental stress has been reported to be the key mediating link to children's behavioral and emotional problems during the pandemic, triggering parents' anxiety and depressive symptoms, which negatively impacted parenting behaviors and parent-child interactions and children's psychological and behavioral difficulties (Romero et al., 2020; Spinelli et al., 2020). It has been argued that COVID-19-stressed parents appear to be less able to understand their children's needs and to respond to them sensitively (Spinelli et al., 2020), but the specific mechanism which accounts for this phenomenon has not been elucidated or tested.

\section{Maternal Mentalization}

Maternal mentalization is a trans-theoretical, multidimensional concept (Schiborr et al., 2013; Sharp \& Fonagy, 2008) which refers to a mother's tendency to treat her child as an individual with an independent mind that is separate from hers (Meins et al., 2001), to see the child as motivated by internal mental states such as desires and feelings (Slade, 2005), and to reflect on her own internal mental states and how they are shaped and changed by interactions with the child (Luyten et al., 2017). Maternal mentalization is thought to promote healthy socioemotional development in children (Zeegers et al., 2017) and has been linked with fewer internalizing and externalizing behaviors among preschoolers (Meins et al., 2013; Smaling et al., 2016). Greater maternal mentalization, particularly mothers' ability to reflect on their own parenting, has been associated with mothers' ability to tolerate stress and self-regulate effectively in the face of high levels of distress and physiological arousal (Rutherford et al., 2015).

Mentalization in general, and in the context of parenting in particular, is vulnerable to stress and arousal. It has been argued that with increasing stress there is a "switch point" at which effortful, reflective mentalization collapses, and reflexive, automated, non-reflective thinking takes over (Fonagy and Luyten 2009). However, individuals with strong mentalization skills are able to recover and return to a mentalizing stance after a brief collapse. In contrast, individuals with poor mentalization may respond to stress by prolonged and extensive mentalization collapses, exacerbating their negative arousal and further interfering with their ability to regain reflective, controlled thinking (Fonagy and Luyten, 2009). Thus, mentalization is considered an asset in the face of stress and distress.

Recently it has been argued that maternal mentalization can serve as a moderator: a protective factor that shields children's development and well-being under adverse circumstances. For instance, higher maternal physiological arousal has been associated with over-controlling behavior 
in mothers and lower reflective functioning in children, but only among mothers with low mentalization, whereas among mothers who had high mentalization skills, no such associations were found (Borelli et al., 2017). Prior to the pandemic, the current researchers showed that maternal mentalization moderated the link between mothers' and fathers' anxiety symptoms and their children's externalizing behaviors (Dollberg et al., 2021). The moderating effect of maternal mentalization in the face of situational arousal and emotional distress can be attributed to the mother's ability to recognize her child's mental and emotional needs (Sharp \& Fonagy, 2008), to be reflective regarding her child's and her own inner experiences and differentiate between the two (Slade, 2005), and consequently to maintain a sensitive parental stance vis-a-vis the child. Because maternal mentalization also involves the ability to self-reflect and appreciate how her (the mother's) own internal mental states may impact the child, it can also assist mothers in modulating their arousal (Borelli et al., 2017; Rutherford et al., 2015), filtering their own negative emotions (such as anxiety and fear), and selfregulating their negative and insensitive parental responses (Borelli et al., 2017; Ensink et al., 2016). These functions may be especially critical during the pandemic, as children need their parents to recognize, support, and address their fears in a calm way and mediate the overwhelming reality to them in a developmentally appropriate, sensitive, and reassuring fashion (Spinelli et al., 2020). A mother who possesses less advanced mentalization skills, or a mother whose mentalization skills have collapsed due to high stress and anxiety (Luyten et al., 2017), may overburden her child with her own anxieties and fears (Asen \& Fonagy, 2012).

In sum, maternal mentalization is considered a parental asset which was shown, prior to the COVID-19 outbreak, to potentially protect children from the negative impact of maternal psychopathology symptoms, and particularly anxiety symptoms. The role of maternal mentalization has not, however, been examined in the context of COVID-19, maternal anxiety symptoms, and child behavior problems. Given that COVID-19 is associated with high levels of maternal anxiety symptoms and children's distress and fears, it is important to examine whether maternal mentalization might shield children from the negative impact of maternal anxiety symptoms during the current pandemic.

\section{The Current Study}

As reviewed above, evidence has accumulated to show an increase in mothers' anxiety symptoms and children's socioemotional and behavioral difficulties during the current, ongoing COVID-19 pandemic. However, not much is known about the specific parenting mechanisms that might contribute to this rise in children's behavioral and emotional problems. Thus, our first objective was to compare reports of maternal anxiety symptoms and children's behavior problems collected from a group of mothers before the pandemic, and a comparable group of mothers assessed shortly after the pandemic's outbreak. Next, given the evidence regarding the mediating role of parents' anxiety in children's behavior problems in general, and particularly in extreme situations, our second goal was to examine whether maternal anxiety symptoms mediated the link between COVID-19 and children's reported internalizing and externalizing behaviors. Finally, emerging data have pinpointed maternal mentalization as a parental resource that can modulate the impact of a stressful event on the child directly, as well as by moderating the indirect negative effect of mothers' anxiety. Thus, our third goal was to test whether maternal mentalization might moderate the direct and indirect links between COVID-19, maternal anxiety symptoms, and children's internalizing and externalizing behaviors.

\section{Study's Hypotheses}

H1: Mothers in the COVID-19 group will report having more anxiety symptoms (H1a) compared to mothers in the pre-COVID-19 group. Mothers in the COVID-19 group will also report their children as having more internalizing and externalizing behaviors (H1b) compared to mothers in the pre-COVID-19 group.

H2: COVID-19 group status will be linked with the child's externalizing (H2a) and internalizing (H2b) behaviors. The associations between COVID-19 group status and child behaviors will be mediated by the mother's anxiety symptoms and moderated by the mother's mentalization skills. Specifically, the direct and indirect links via the mother's anxiety symptoms will be stronger for mothers with weaker mentalization.

\section{Method}

\section{Participants}

We tested our hypotheses among a sample comprising 140 Israeli mothers who had a child between 3-6 years of age. The sample consisted of a COVID-19 group $(n=53)$, recruited during the first wave of the pandemic's outbreak, and a pre-COVID-19 group $(n=87)$, of a similar background, recruited prior to the pandemic. Inclusion criteria for both groups were good reported physical health of mothers and children, absence of major developmental difficulties in the child (that required special education), and mothers cohabiting with a spouse/child's father. For both groups, only mothers who completed all the measures were 
included in the current analysis. Mothers' mean age for the entire sample was $35.55(S D=4.59)$. The overall sample consisted of $70(50 \%)$ boys, and the majority of them were firstborn children $(91,64 \%)$. Children's age for the overall sample averaged $4.17(S D=0.87)$. About half of the mothers in the overall sample $(72,50 \%)$ reported an above-average household income based on Israeli standards, another 31\% (44) reported an average household income, and a minority $(25,17.6 \%)$ reported a below-average income. Table 1 presents the demographics of the two groups as well as the comparison among them.

\section{Procedure}

Recruitment for the COVID-19 group was carried out in the midst of the pandemic's outbreak and during an enforced quarantine in Israel (from mid-March until the end of April 2020). During these months, advertisements were posted on parents' social networks, and the link to the questionnaire was spread in a snowball-like fashion. Due to the quarantine at that time, the entire procedure was administered online, including the mind-mindedness interview. Data collection ended when the quarantine ended and the school system reopened (with certain restrictions). The study was approved by the Acadmic College of Tel Aviv Yaffo's Ethics Committee (ref. 2020079). Mothers from the pre-COVID-19 group were similarly recruited through posts on social networks and the snowball method. Mothers completed online questionnaires and were interviewed and audiotaped with the mind-mindedness assessment procedure during a home visit. Mothers from both groups were asked to consider only one of their children in the specified age range when completing the questionnaires. All participants signed an informed consent prior to participation, participation was anonymous and voluntary, and there was no reward or compensation for participation.

\section{Measures}

Mothers' Anxiety Symptomatology We used the 6-item anxiety subscale of the Brief Symptom Index (BSI; Derogatis, 1993 ) to assess each mother's reported anxiety symptoms. The BSI is a widely used, psychometrically validated, reliable self-report questionnaire (Derogatis \& Melisaratos, 1983), comprising 53 items that measure distress. Respondents were asked to answer questions such as "Over the last month, to what extent did you feel fearful?" on a 5-point Likert-type scale, ranging from 0 (not at all) to 4 (extremely). Higher scores reflect greater psychopathology symtpoms. Internal consistency of the anxiety subscale in the current study was found to be good for both the pre-COVID-19 and the COVID-19 group ( $\alpha=0.77,0.78$ respectively).

Maternal Mentalization We utilized Meins and colleagues' (see Meins \& Fernyhough, 2010) mind-mindedness (MM) procedure to assess mothers' mentalization representational
Table 1 Demographics of the COVID-19 and Pre-COVID-19 groups

\begin{tabular}{|c|c|c|c|c|}
\hline & $\begin{array}{l}\text { COVID-19 Group } \\
(n=53)\end{array}$ & $\begin{array}{l}\text { Pre-COVID-19 Group } \\
(n=87)\end{array}$ & $t / \chi^{2}$ & $P$ \\
\hline & $\mathrm{M}(\mathrm{SD})$ & $\mathrm{M}(\mathrm{SD})$ & & \\
\hline Child's Age (years) & $4.53(.82)$ & $3.96(.84)$ & -4.01 & $<.001$ \\
\hline Mother's Age (years) & $35.47(4.88)$ & $35.59(4.43)$ & & n.s \\
\hline \multirow[t]{2}{*}{ Number of children in the family } & $2.15(.60)$ & $2.24(.85)$ & & \\
\hline & $\mathrm{N}(\%)$ & $\mathrm{N}(\%)$ & & \\
\hline Child's Gender: Boy & $28(53 \%)$ & $43(49 \%)$ & & n.s \\
\hline Mother's education: & & & & n.s \\
\hline High school and below & $3(6 \%)$ & $2(2 \%)$ & & \\
\hline Post High school diploma & $3(6 \%)$ & $5(6 \%)$ & & \\
\hline $\mathrm{BA}$ & $15(28 \%)$ & $38(44 \%)$ & & \\
\hline MA and above & $32(60 \%)$ & $40(46 \%)$ & & \\
\hline Unknown & 0 & $2(2 \%)$ & & \\
\hline \multicolumn{5}{|l|}{ Child's birth order: } \\
\hline First & $39(73 \%)$ & $52(60 \%)$ & & n.s \\
\hline \multicolumn{5}{|l|}{ Education setting: } \\
\hline Public & $53(100 \%)$ & $54(62 \%)$ & 27.66 & $<.001$ \\
\hline \multicolumn{5}{|l|}{ Household Income: } \\
\hline Below-average & $3(6 \%)$ & $21(24 \%)$ & 12.44 & $<.01$ \\
\hline Average & $24(45 \%)$ & $20(23 \%)$ & & \\
\hline Above-average & $26(49 \%)$ & $46(53 \%)$ & & \\
\hline
\end{tabular}


skills. Mothers were asked: "Can you describe [child] for me?" The mothers were told that there were no right or wrong answers and that they could describe their children in any way they wished. For mothers in the pre-COVID-19 group, the mind-mindedness procedure was conducted by interviews that were recorded and transcribed verbatim prior to coding. Mothers in the COVID-19 group were asked to write online their response to "Can you describe [child] for me?" For both groups, coding consisted of identifying comments that included internal-state terms referring to the child's mind, such as references to the child's wishes, desires, mental states (e.g., thoughts, knowledge, interests), mental processes (e.g., recognition, remembering, decisionmaking), emotions, and attempts to manipulate people's beliefs (e.g., joking, teasing). Next, the rate of these statements (mind-mindedness statements) out of the total number of statements provided by the mother was calculated, and this figure represented the mother's representational mentalization (mind-mindedness) level. Two graduate students trained by the first author coded the material. Interrater agreement during coding was tested on $10 \%$ of randomly selected interviews in each group and was good for both (pre-COVID-19 kappa $=0.85$, COVID-19 kappa $=0.94$ ).

Children's Behavior Problems The Child Behavioral Checklist (CBCL; Achenbach \& Rescorla, 2000) for children (1.5- 5 years old version) was used to evaluate the child's internalizing and externalizing behaviors as perceived by the mother. The CBCL is a widely used 99 -item questionnaire describing child behavior during the past months. Items are presented on a 3-point Likert-type scale, with $0=n o t$ true, $1=$ somewhat or sometimes true, and $2=$ very or often true. Item examples include "afraid to try new things" and "can't sit still, is restless, or hyperactive." The CBCL produces two clusters: the internalizing behavior cluster and the externalizing behavior cluster. In the current study both clusters demonstrated good internal consistency in the preCOVID-19 group (internalizing: $\alpha=0.82$; externalizing: $\alpha=0.86$ ) as well as the COVID-19 group (internalizing: $\alpha=0.88$; externalizing: $\alpha=0.89$ ).

\section{Data Analysis Strategy}

We first compared the COVID-19 and pre-COVID-19 groups in terms of demographics. We then computed Pearson's $r$ correlations to investigate zero-order associations among the study's variables, and between them and the participants' demographics. To test H1 we used ANCOVA. To test H2, we used the PROCESS version 3.0 macro (Hayes, 2018) model 59 for testing moderation and mediation effects with 5,000 bias-corrected bootstrap samples (Hayes, 2018). PROCESS allowed us to combine mediation and moderation within a single model and estimate the conditional and unconditional direct and indirect effects. We ran the analyses for the child's externalizing and internalizing behaviors separately, resulting in two models. In each model we tested the direct and indirect links between COVID-19 group status (pre-COVID-19 vs. COVID-19) and child's externalizing/ internalizing behaviors, mediated via mothers' anxiety and moderated by mothers' mind-mindedness, while controlling for possible demographic confounders. We considered the effects significant at $p<0.05$. When the $95 \%$ confidence interval included 0 , we inferred a significant indirect effect at the 0.05 level. Given the relatively small sample size, we applied the bias-corrected bootstrap samples method (Hayes \& Preacher, 2013). All analyses were performed with SPSS version 25 (IBM Corp, 2017).

\section{Results}

First, we compared the COVID-19 and pre-COVID-19 groups in terms of demographics. The results are presented in Table 1. Accordingly, we found that children in the preCOVID-19 group were younger and, relatedly, tended to attend private (rather than public) schools. Furthermore, "below-average" household income was more prevalent in the pre-COVID-19 group than in the COVID-19 group. Next, we examined zero-order correlations between the variable in which the groups differed (i.e., child's age, education setting, and household income) and the study's variables. This procedure revealed significant correlations between "child's age" $(r=0.31, p<0.001)$ and "child's education setting" $(r=-0.25 . p<0.01)$ and mothers' mind-mindedness, so that mothers of older children and those attending public schools (and therefore likely to be older) displayed higher mind-mindedness. Household income did not correlate with any of the study's variables. Therefore "child's age" and "education setting" were included in the analyses where mind-mindedness was tested.

Next, we examined intercorrelations between the study's variables for the entire sample and within each group. Table 2 presents the study's variables' means, SDs, and intercorrelations for the entire sample and for each group. As shown in Table 2, we found strong positive correlations between the mothers' anxiety symptom scores and their reports of their children's internalizing and externalizing behaviors for the entire sample and within each group. Maternal mentalization did not correlate with either maternal anxiety or child behavior problems.

In support of $\mathrm{H} 1$, the multivariate analysis showed an overall significant between-group difference, $F$ (3, $136)=6.30, p<0.001, \eta^{2}=0.12$. Univariate analysis revealed the expected differences between the groups, so that mothers in the COVID-19 group reported significantly more anxiety symptoms $(\mathrm{H} 1 \mathrm{a}), F(1,138)=18.97, p<0.001$, 
Table 2 Means, SDs, and intercorrelations between the study's variables for the entire sample $(\mathrm{N}=140)$ and by group

\begin{tabular}{|c|c|c|c|c|c|}
\hline & M & SD & $\begin{array}{l}\text { Mother's } \\
\text { Mind-Minded- } \\
\text { ness }\end{array}$ & $\begin{array}{l}\text { Child's } \\
\text { Externalizing } \\
\text { Behaviors }\end{array}$ & $\begin{array}{l}\text { Child's } \\
\text { Internalizing } \\
\text { Behaviors }\end{array}$ \\
\hline Mother's Anxiety & 1.80 & .55 & .15 & $.55^{* *}$ & $.47^{* *}$ \\
\hline Mother's Mind-Mindedness & .51 & .20 & - & -.01 & .02 \\
\hline Child's Externalizing Behaviors & 32 & 6.33 & - & - & $.68^{* *}$ \\
\hline Child's Internalizing Behaviors & 42.26 & 6.60 & - & - & - \\
\hline \multicolumn{6}{|l|}{ COVID-19 Group $(n=53)$} \\
\hline Mother's Anxiety & 2.04 & .60 & -.06 & $.52^{* *}$ & $.56 * *$ \\
\hline Mother's Mind-Mindedness & .60 & .20 & - & -.05 & -.06 \\
\hline Child's Externalizing Behaviors & 33.45 & 7.19 & - & - & $.67^{* *}$ \\
\hline Child's Internalizing Behaviors & 43.77 & 7.39 & - & - & - \\
\hline \multicolumn{6}{|l|}{ Pre-COVID-19 Group $(n=87)$} \\
\hline Mother's Anxiety & 1.65 & .46 & .09 & $.53^{* *}$ & $.33^{* *}$ \\
\hline Mother's Mind-Mindedness & .46 & .17 & - & -.11 & -.03 \\
\hline Child's Externalizing Behaviors & 31.11 & 5.60 & - & - & $.64^{* *}$ \\
\hline Child's Internalizing Behaviors & 41.33 & 5.92 & - & - & - \\
\hline
\end{tabular}

$\eta^{2}=0.12$, and more child internalizing, $F(1,138)=4.63$, $p<0.05, \eta^{2}=0.03$, and externalizing behaviors (H1b), $F$ (1, $138)=4.62, p<0.05, \eta^{2}=0.03$, compared to mothers in the pre-COVID-19 group. Thus, hypotheses H1a and H1b were fully supported.

We tested H2 with two models, one predicting child's externalizing and the other predicting child's internalizing behaviors. Power analysis using $\mathrm{G}^{*}$ Power3.1.9.2 software showed that the sample size was sufficient for detecting a medium-sized $\left(f^{2}=0.35\right)$ mediation-moderation effect with a probability of almost $100 \%$. As hypothesized, the model predicting child's externalizing behaviors (H2a) was significant, $F(7,132)=10.12, p<0.001 R^{2}=0.35$. Figure 1 presents the direct and the mediated moderated links between the variables. The model was tested with child's age and education setting as covariates; however, for the sake of visual clarity, these covariates are not presented in the figure. Accordingly, mothers' anxiety fully mediated the

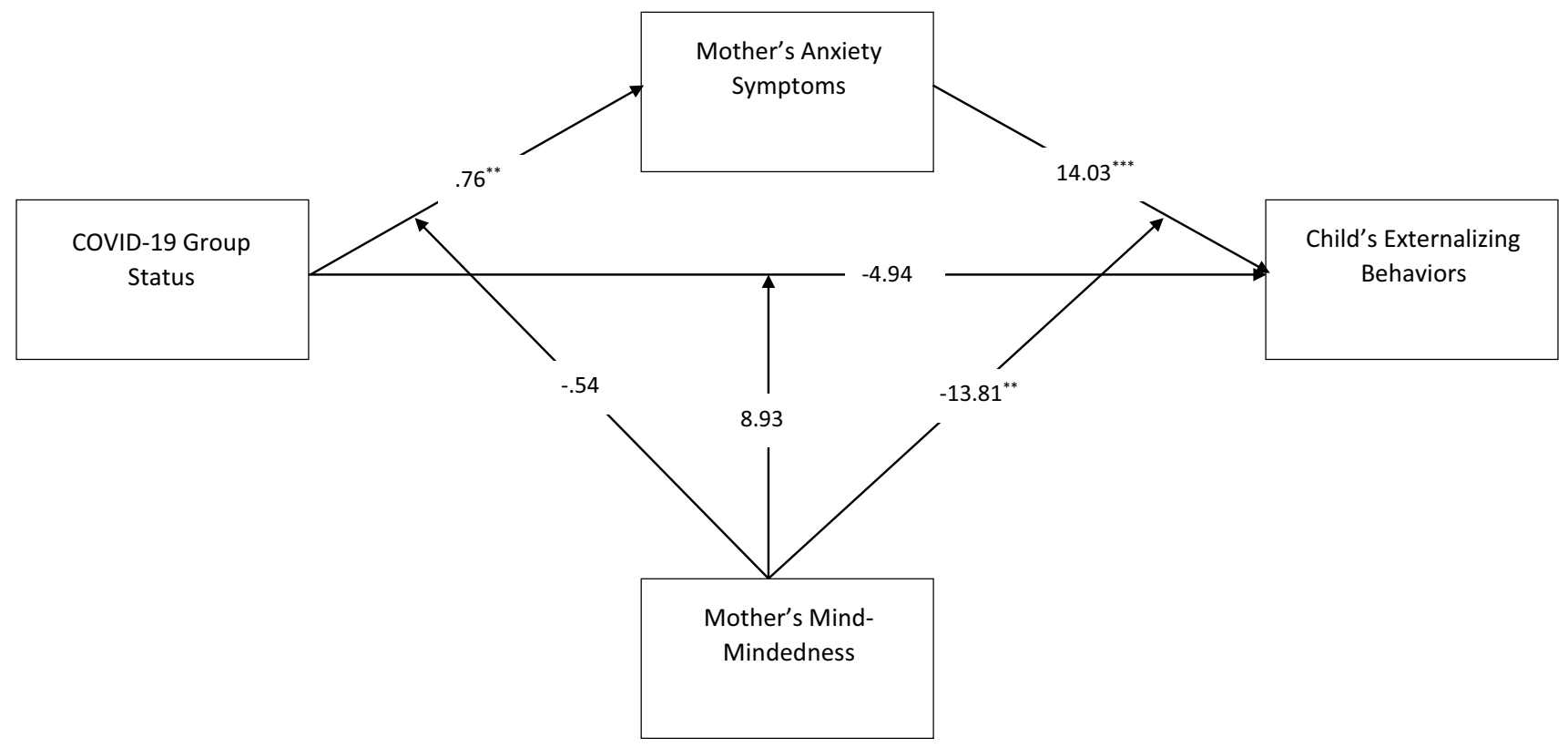

Fig. 1 Mediated moderation model of COVID-19 group status predicting child's externalizing behaviors via mother's anxiety symptoms moderated by mother's mind-mindedness. Note: Numbers on lines are unstandardized path coefficients; ${ }^{* *} p<.01,{ }^{* * *} p<.001$ 
link between COVID-19 group status and child's externalizing behaviors. Furthermore, a significant interaction effect between mothers' mind-mindedness and mothers' anxiety was found. Indirect links were tested at three points of mindmindedness: low mind-mindedness (1 SD below average), medium mind-mindedness (average), and high mind-mindedness ( $1 \mathrm{SD}$ above average). Table 3 presents the conditional indirect effects at all three points. As can be seen in Table 3, significant indirect effects were found at all three points. Pairwise contrast tests showed that the differences in effects between the three points of mind-mindedness were significant. Figure 2 provides a visual demonstration of the conditional effects of the focal predictor on the values of the moderator as produced by the PROCESS script. As indicated in Table 3 and visually presented in Fig. 2, the strongest association between mother's anxiety symptoms and child's

Table 3 Conditional Indirect Effects of COVID-19 Group Status Mediated by Mother's Anxiety Symptoms on Child's Externalizing and Internalizing Behaviors

\begin{tabular}{llll}
\hline & $B$ & $S E-B$ & LLCI,ULCI $^{1}$ \\
\hline Child's Externalizing Behaviors & & & \\
Low Mind-Mindedness & 5.63 & 2.05 & $2.23,10.13$ \\
Medium Mind-Mindedness & 3.32 & 1.03 & $1.54,5.50$ \\
High Mind-Mindedness & 1.58 & .85 & $.26,3.50$ \\
Child's Internalizing Behaviors & & & \\
Low Mind-Mindedness & 4.57 & 2.34 & $.96,9.69$ \\
Medium Mind-Mindedness & 2.83 & 1.21 & $.94,5.56$ \\
High Mind-Mindedness & 1.50 & .92 & $.19,3.77$ \\
\hline
\end{tabular}

Low Mind-Mindedness = 1SD above Mean; Medium Mind-Mindedness $=$ Mean; High Mind-Mindedness $=1$ SD above Mean

${ }^{1}$ Level of confidence intervals is $95 \%$ with 5,000 bootstrap samples externalizing behaviors was when mother's mind-mindedness was low, whereas when mother's mind-mindedness was higher, the association was weaker. In sum, hypothesis $\mathrm{H} 2 \mathrm{a}$ was fully supported.

The model predicting child's internalizing behaviors (H2b) was also significant, $F(7,132)=6.15, p<0.001$ $R^{2}=0.25$. Mothers' anxiety similarly fully mediated the link between COVID-19 group status and child's internalizing behaviors at all points of mothers' mind-mindedness. Table 3 presents the conditional indirect effects at all three points. Accordingly, significant indirect effects were found at all three points of mothers' mind-mindedness. However, contrary to our hypothesis, no interaction effect of mothers' mind-mindedness on either the direct or indirect links was found. Thus, hypothesis H2b was only partially supported.

\section{Discussion}

Our findings join a rapidly emerging and greatly needed body of research documenting the increase in adult and children's psychopathology symptoms in response to the current pandemic. Specifically, our study showed that during COVID-19, mothers of preschool children experienced more anxiety symptoms and perceived their children as having more externalizing and internalizing behaviors than did mothers of children of similar ages and background who were interviewed before the COVID-19 outbreak. Moreover, our findings indicated that, as hypothesized, mothers' anxiety symptoms mediated the effect of the pandemic on children's behavior problems so that the higher mothers' anxiety was, the more externalizing and internalizing behaviors were reported by the mothers about their children. Finally, the
Fig. 2 Associations between mother's anxiety symptoms and child's externalizing behaviors by mother's mindmindedness level. Note: Low Mind-Mindedness $=1 \mathrm{SD}$ above Mean; Medium Mind-Mindedness $=$ Mean; High Mind-Mindedness $=1$ SD above Mean

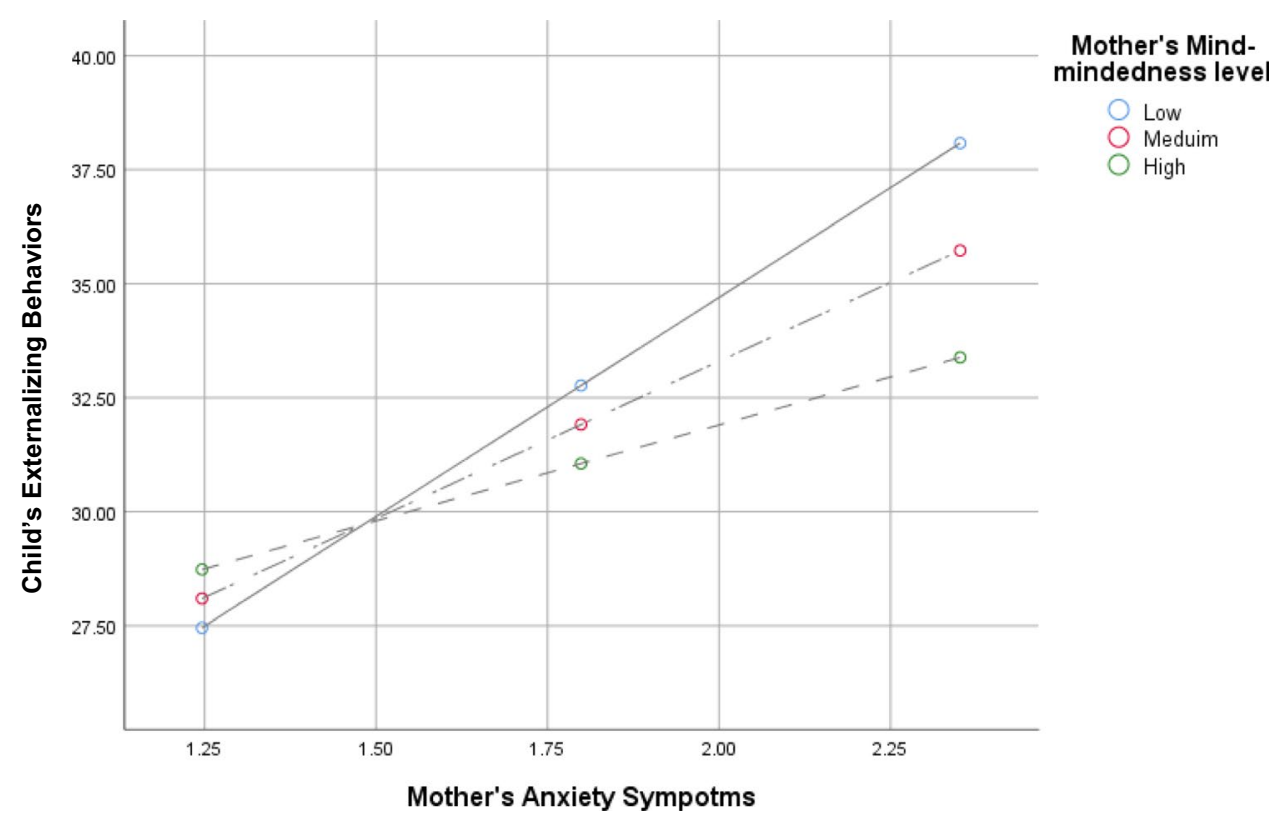


findings supported our hypothesis that mothers' mind-mindedness moderated the association between the pandemic and children's externalizing behaviors. Specifically, when mothers showed higher mentalization skills (higher mindmindedness), the indirect effect of anxiety symptoms on the link between COVID-19 and child's externalizing behaviors was weaker than when mothers showed lower mentalization skills (lower mind-mindedness).

The finding regarding higher rates of anxiety symptoms among mothers and more reports of behavior problems in their children during COVID-19 compared to reports by mothers interviewed prior to the pandemic is consistent with mounting evidence from around the world documenting the negative socioemotional impact of the pandemic on adults and particularly on mothers (e.g., Dubey et al., 2020) and on their children (Davenport et al., 2020; Domínguez-Álvarez et al., 2020; Jiao et al., 2020; Malhotra et al., 2020; Orgilés et al., 2020). The increase in mothers' anxiety symptoms and children's socioemotional and behavioral difficulties are understandable, given the objective and subjective risks associated with the pandemic, and the health, economic, and social crises accompanying it. Of note is the fact that our data were collected shortly after the World Health Organization (WHO) declared that the COVID-19 outbreak constituted a pandemic, leading Israel, like other countries, to enforce a countrywide quarantine, shutting down schools, workplaces, playgrounds, and recreation sites, and limiting social and extended family gatherings. Moreover, initially the medical establishment and the public had a very limited understanding of the disease, the mechanisms by which it spread, and the ways in which to manage and treat it. The ambiguity and sense of threat that surrounded the pandemic may have led mothers, and particularly mothers of young children, to feel overwhelmed, fearful, and helpless, all possible contributors to the increased anxiety among mothers and the socioemotional difficulties among their children (Spielberger, 2013).

Accumulating data indicate that children's malevolent or pathological responses to external crises, and particularly the responses of younger children, are closely tied to their caregivers' reactions to the crises (Juth et al., 2015). Our findings provide further support for this argument by showing that, as hypothesized, mothers' anxiety symptoms mediated the negative link between COVID-19 and children's elevated internalizing and externalizing behaviors. In other words, it was not the pandemic itself that accounted for the increase in children's externalizing and internalizing behaviors. Rather, the pandemic seemed to lead to mothers' experience of elevated anxiety symptoms, which, in turn, seemed to increase the risk of more externalizing and internalizing behaviors in their children. Supporting this idea, recent reports have indicated that mothers' anxiety and stress during the pandemic has interfered with parenting (Romero et al., 2020), leading mothers to demonstrate more hostile and coercive parenting (Chung et al., 2020) which, in turn, may increase the risk of more externalizing and internalizing behaviors among their children.

Consistent with our hypothesis, mothers' mentalization skills, operationalized via the mind-mindedness construct, moderated the negative link between their anxiety symptoms and their children's externalizing behaviors. Specifically, as hypothesized, mothers who demonstrated lower mentalization skills and higher anxiety symptoms also reported more child externalizing behaviors. In contrast, when the mother demonstrated better mentalization skills, she also reported less anxiety symptoms and less externalizing behaviors in her child. Of note is the fact that maternal mentalization was unrelated to mothers' anxiety symptoms; it also did not moderate the direct link between COVID-19 group status and child's externalizing and internalizing behaviors. Taken together, these findings suggest that although maternal mentalization may not spare mothers from experiencing anxiety or undo the negative effects of COVID-19 on mothers and children, it can reduce the risk of children developing behavior problems despite the potentially negative impact upon them of their mothers' anxiety symptoms. Given these findings and what we know about how parental mentalization works, we can suggest, albeit tentatively, specific mechanisms by which mentalization may exert its buffering effect on children during the current pandemic. Accordingly, higher maternal mentalization helps the mother to "step into her child's shoes" (Meins et al., 2001), differentiate between her own and her child's mind (Sharp \& Fonagy, 2008), and appreciate her child's limited developmental capacity to comprehend complex situations (Slade, 2005). This understanding can lead mothers with higher mentalization skills to appreciate the potentially overwhelming effect of their own anxiety on their young children and therefore try to conceal and modulate it. In terms of the current study's findings, mothers in the COVID-19 group with higher mentalization skills may have tried and been successful in moderating their own anxiety when interacting with their young children and, by doing so, provided their children with a sense of safety and assurance. In contrast, mothers with lower mentalization skills may have shared their worries with their children, overburdening and confusing them and thus increasing the risk of behavior problems in their children. These mothers may have discussed frightening information in the presence of the children or overwhelmed them with media reports, failing to appreciate how these actions may have affected the children emotionally. This explanation is in accord with earlier findings reported prior to the pandemic, indicating that mothers who are characterized by higher mentalization skills are better at recognizing and modulating their negative emotions (Ensink et al., 2016) and physiological arousal (Rutherford et al., 2015) when stressed. Furthermore, 
mothers with higher mentalization skills may be more tolerant and accepting of their children's negative behaviors, and particularly externalizing behaviors, because they look for and recognize the inner experiences that lie beneath the behaviors and act as affect regulators for their children (Gergely et al., 2002). In contrast, mothers with lower mentalization skills may fail to understand the underlying motives beneath their children's behavior, particularly when it comes to disturbing behaviors. Indeed, recent reports have indicated that during the pandemic, many children have reacted with more problematic behaviors (Jiao et al., 2020; Orgilés et al, 2020; Romero et al., 2020). Thus, mothers with weaker mentalization skills may have reacted more negatively to their children's externalizing behaviors, failing to anticipate, contain, and understand them, which may have further increased their own distress, sense of helplessness, and anxiety. Thus, a vicious circle may emerge in which mothers characterized by more anxiety symptoms and lower mentalization skills may face more disturbing externalizing behaviors in their children which, in turn, may further add to the mother's already high anxiety symptoms.

Unexpectedly, we did not find a moderating effect of maternal mentalization in regard to internalizing behaviors, despite a mediating effect of maternal anxiety symptoms on the link between COVID-19 and child's internalizing behaviors. These results accord with those of a Colonnesi and colleagues' (2019) study, in which they were unable to find the predicted association between parental mindmindedness and preschoolers' internalizing behaviors. The authors attributed the lack of significant findings to the fact that internalized behaviors are less apparent behaviorally in preschoolers and are therefore less visible to parents. Similarly, we would argue that the kind of symptomatology associated with internalizing behaviors (i.e., fears, withdrawal, and avoidance) may be perceived by mothers, regardless of their mentalization skills, as more normative and acceptable during the preschool years in general and in the context of the pandemic in particular. Finally, the lack of a significant moderation effect may be due to the size of the sample and limited statistical power.

\section{Strengths, Limitations, and Future Studies}

Our study's strengths lie in comparing - via self-report questionnaires and an open-ended, semi-structured measurement - mothers' and children's socioemotional functioning before and during the COVID-19 outbreak. In addition, it offers and tests a conceptual model to explain the increase as well as variations in COVID19-related symptomatology among mothers and children. Its value lies in the comparison of two groups of mothers and children of similar backgrounds assessed by the same research tools during two significantly different times: before and during the pandemic. However, there were several limitations, including a relatively modest sample size, concurrent data collection in each group, and a reliance on mothers as a source of information. These factors limited our ability to detect additional effects and to determine causality. For example, the increase in mothers' reports of their own anxiety symptoms and their children's elevated behavior problems may at least in part have been a reflection of the media's extensive reports of such phenomena, potentially priming mothers to have a heightened or inaccurate awareness of them, rather than being a reflection of a real, objective increase in such behaviors. Additional limitations included focusing on heterosexual mothers who share household and childcare responsibilities with a spouse, and collecting data electronically, limiting the generalizability of the findings to other, more at-risk populations. Furthermore, the study's procedure did not allow us to assess the contribution of fathers' and/or dyadic influences in the context of children and families coping with the pandemic. Finally, given home confinement and social distancing regulations during the recruitment of the COVID-19 group, all data collection was carried out via the internet. In contrast, in the pre-COVID-19 group, the mind-mindedness interview procedure was conducted in person. This, as well as other group differences might better explain the current findings. Future studies should use longitudinal designs, following parents (both mothers and fathers) who were recruited prior to the pandemic and assessing relevant parental reports during the pandemic and in the future, after the pandemic is hopefully contained. Future studies should also expand the assessment procedures, assessing children's and parents' symptoms of psychopathology and socioemotional distress via other sources (e.g., teachers' reports and clinical interviews). Finally, we used the mind-mindedness construct to assess maternal mentalization. The mind-mindedness construct assesses a particular aspect of mentalization, namely the mother's tendency to view her child as a mental agent. Thus, the role of other aspects of maternal mentalization such as parental reflective functioning could not be addressed by this study. Moreover, it has been argued that mothers who demonstrate high off-line, interview-derived maternal mentalization skills do not necessarily demonstrate the same skills when interacting with their children directly (Shai et al., 2017). Thus, future studies should use other methods of assessing parental mentalization to fully understand its many dimensions.

\section{Conclusions and Clinical Implications}

The COVID-19 pandemic is an ongoing, global crisis with extensive health, economic, social, and psychological implications for children and their parents that are only beginning to be explored and understood. Our study's results underscore the risk associated with maternal anxiety symptomatology for 
children's behavior problems when mothers are overwhelmed and fail to contain their distress. On a more optimistic note, the findings also indicate that mothers' mentalization skills may serve as a source of resilience, protecting children from the risk associated with maternal anxiety. This point is especially important at the current time, when mothers are called on to help their children overcome the emotional and behavioral difficulties linked to the pandemic and its psychological sequelae. Drawing on our findings, we can argue, albeit tentatively, that supporting mothers by helping them to manage and contain their anxiety symptoms can help decrease signs of psychopathology in both mothers and their young children. Thus, encouraging mothers to reduce their anxiety levels by making time for themselves to exercise, practice relaxation and mindfulness techniques, and maintain social connections, even if only remotely, is crucial. Mothers might also benefit from developmental guidance and psychoeducation regarding young children's reactions to threats and can learn techniques to better manage children's negative reactions to the current pandemic. Furthermore, the findings provide support for our assertion that maternal mentalization is an important parental resource. There are several evidence-based therapeutic interventions that work to enhance parental mentalization during the preschool years (e.g., Huber et al., 2015; Ross et al., 2020; Suchman et al., 2010). These may be adapted and utilized as preventive and therapeutic interventions to enhance maternal mentalization among mothers/parents coping with the pandemic and its aftermath.

Acknowledgements The authors wish to thank Ravit Bachar and Inbar Ben Moshe for their assistance in data collection and coding. We also wish to thank the mothers who volunteered to participate in the study.

Authors' Contribution The manuscript is based on original data collected by our team. We are the primary researchers in this project and have co-authored the current submitted manuscript.

Data Availability The datasets generated during and/or analyzed during the current study are available from the corresponding author upon reasonable request.

\section{Declarations}

Conflict of Interest The authors declare that they have no conflicts of interest.

Compliance with Ethical Standards The study was approved by the institutional ethics committee and have been performed in accordance with the ethical standards of the Helsinki declaration. Participation in the study was voluntary. All participants signed an informed consent prior to participation.

\section{References}

Achenbach, T., \& Rescorla, L. (2000). ASEBA preschool forms \& profiles. Burlington, VT: University of Vermont, Research Center for Children, Youth and Families.

Asen, E., \& Fonagy, P. (2012). Mentalization-based therapeutic interventions for families. Journal of Family Therapy, 34(4), 347-370.

Brooks, S. K., Webster, R. K., Smith, L. E., Woodland, L., Wessely, S., Greenberg, N., \& Rubin, G. J. (2020). The psychological impact of quarantine and how to reduce it: rapid review of the evidence. The Lancet., 395, 912-20.

Behrendt, H. F., Wade, M., Bayet, L., Nelson, C. A., \& Enlow, M. B. (2019). Pathways to social-emotional functioning in the preschool period: The role of child temperament and maternal anxiety in boys and girls. Development and Psychopathology, 1-14.

Borelli, J. L., Hong, K., Rasmussen, H. F., \& Smiley, P. A. (2017). Reflective functioning, physiological reactivity, and overcontrol in mothers: Links with school-aged children's reflective functioning. Developmental Psychology, 53(9), 1680-1693.

Chung, S. K. G., Lanier, P., \& Wong, P. (2020). Mediating effects of parental stress on harsh parenting and parent-child relationship during Coronavirus (COVID-19) pandemic in Singaporel https:// doi.org/10.1007/s10896-020-00200-1

Cobham, V. E., McDermott, B., Haslam, D., \& Sanders, M. R. (2016). The role of parents, parenting and the family environment in children's post-disaster mental health. Current Psychiatry Reports, 18(6), 53.

Colonnesi, C., Zeegers, M. A., Majdandžić, M., van Steensel, F. J., \& Bögels, S. M. (2019). Fathers' and mothers' early mind-mindedness predicts social competence and behavior problems in childhood. Journal of Abnormal Child Psychology, 47(9), 1421-1435.

Coyne, L. W., Gould, E. R., Grimaldi, M., Wilson, K. G., Baffuto, G., \& Biglan, A. (2020). First things first: Parent psychological flexibility and self-compassion during COVID- 19. Behavior Analysis in Practice, 1.

CBS (2020). Israeli Central Bureau of Statistics Publication, Public Use File (PUF): https://www.cbs.gov.il/he/Statistical/statistical182-corona.pdf.

Davenport, M. H., Meyer, S., Meah, V. L., Strynadka, M. C., \& Khurana, R. (2020). Moms are not ok: COVID-19 and maternal mental health. Frontiers in Global Women's Health, 1,1 .

Derogatis, L. R. (1993). BSI Brief Symptom Inventory: Administration, scoring, and procedure manual (4th ed.). Minneapolis, MN: National Computer Systems.

Derogatis, L. R., \& Melisaratos, N. (1983). The Brief Symptom Inventory: An introductory report. Psychological Medicine, 13, 595-605.

Dollberg, D. G., Hanetz-Gamliel, K., \& Levy, S. (2021). Mediating and moderating links between coparenting, parental mentalization, parents' anxiety, and children's behavior problems. Journal of Family Psychology, 35(3), 324.

Domínguez-Álvarez, B., López-Romero, L., Isdahl-Troye, A., GómezFraguela, J. A., \& Romero, E. (2020). Children Coping, Contextual Risk and Their Interplay During the COVID-19 Pandemic: A Spanish Case. Frontiers in Psychology, 11, 3427.

Dubey, P., Reddy, S., Manuel, S., \& Dwivedi, A. K. (2020). Maternal and neonatal characteristics and outcomes among COVID-19 infected women: An updated systematic review and meta-analysis. European Journal of Obstetrics \& Gynecology and Reproductive Biology.

Ensink, K., Normandin, L., Plamondon, A., Berthelot, N., \& Fonagy, P. (2016). Intergenerational pathways from reflective functioning to infant attachment through parenting. Canadian Journal of Behavioural Science / Revue Canadienne Des Sciences Du Comportement, 48(1), 9-18. 
Fonagy, P., \& Luyten, P. (2009). A developmental, mentalizationbased approach to the understanding and treatment of borderline personality disorder. Developmental Psychopathology, 21(4), $1355-1381$.

Gavin, B., Lyne, J., \& McNicholas, F. (2020). Mental health and the COVID-19 pandemic. Irish Journal of Psychological Medicine, 37(3), 156-158.

Gergely, G., Fonagy, P., Jurist, E., \& Target, M. (2002). Affect regulation, mentalization, and the development of the self. International Journal of Psychoanalysis, 77, 217-234.

Ghosh, R., Dubey, M. J., Chatterjee, S., \& Dubey, S. (2020). Impact of COVID-19 on children: Special focus on psychosocial aspect. Education, 31, 34.

Gibler, R. C., Kalomiris, A. E., \& Kiel, E. J. (2018). Paternal anxiety in relation to toddler anxiety: The mediating role of maternal behavior. Child Psychiatry \& Human Development, 49(4), 512-522.

Golberstein, E., Wen, H., \& Miller, B. F. (2020). Coronavirus disease 2019 (COVID-19) and mental health for children and adolescents. JAMA pediatrics.

Hanetz-Gamliel, K., Dollberg, D., \& Levy, S. (2018). Relations between parents' anxiety symptoms, marital quality and Internalizing and Externalizing behaviors in preschoolers. Journal of Child and Family Studies, 27(12), 3952-3963.

Hayes, A. F. (2018). Introduction to mediation, moderation, and conditional process analysis second edition: A regression-based approach. Ebook The Guilford Press. Google Scholar.

Hayes, A. F., \& Preacher, K. J. (2013). Conditional process modeling: Using structural equation modeling to examine contingent causal processeśl. In G. R. Hancock \& R. O. Mueller (Eds.), Quantitative methods in education and the behavioral sciences: Issues, research, and teaching. Structural equation modeling: A second course (pp. 219-266). Charlotte, NC: IAP Information Age Publishing.

Holmes, E. A., O'connor, R. C., Perry, V. H., Tracey, I., Wessely, S., Arseneault, L., ... \& Bullmore, E. Multidisciplinary research priorities for the COVID-19 pandemic: a call for action for mental health science. Lancet Psychiatry. 2020 Jun; 7 (6): 547-560. https://doi.org/10.1016/S2215-0366(20)30168-1.]

Huber, A., McMahon, C. A., \& Sweller, N. (2015). Efficacy of the 20-week circle of security intervention: Changes in caregiver reflective functioning, representations, and child attachment in an Australian clinical sample. Infant Mental Health Journal, $36(6), 556-574$.

Imran, N., Aamer, I., Sharif, M. I., Bodla, Z. H., \& Naveed, S. (2020). Psychological burden of quarantine in children and adolescents: A rapid systematic review and proposed solutions. Pakistan Journal of Medical Sciences, 36(5), 1106.

IBM Corp (2017). IBM SPSS Statistics for Windows, Version 25.0. Armonk, NY: IBM Corp.

Jiao, W. Y., Wang, L. N., Liu, J., Fang, S. F., Jiao, F. Y., PettoelloMantovani, M., et al. (2020). Behavioral and emotional disorders in children during the COVID-19 epidemic. Journal of Pediatrics, 221, 264-266.e1. https://doi.org/10.1016/j.jpeds. 2020.03.013

Juth, V., Silver, R. C., Seyle, D. C., Widyatmoko, C. S., \& Tan, E. T. (2015). Post-disaster mental health among parent-child dyads after a major earthquake in Indonesia. Journal of Abnormal Child Psychology, 43(7), 1309-1318. https://doi.org/10.1007/ s10802-015-0009-8

Lei, L., Huang, X., Zhang, S., Yang, J., Yang, L., \& Xu, M. (2020). Comparison of prevalence and associated factors of anxiety and depression among people affected by versus people unaffected by quarantine during the COVID-19 epidemic in southwestern China. Medical Science Monitor: International Medical Journal of Experimental and Clinical Research, 26, e924609-e924611.
Luo, M., Guo, L., Yu, M., \& Wang, H. (2020). The Psychological and Mental Impact of Coronavirus Disease 2019 (COVID-19) on Medical Staff and General Public-A Systematic Review and Meta-analysis. Psychiatry Research, 113190.

Luyten, P., Mayes, L. C., Nijssens, L., \& Fonagy, P. (2017). The parental reflective functioning questionnaire: Development and preliminary validation. PloS One, 12(5), e0176218.

Malhotra, C., Chaudhry, I., Ozdemir, S., Teo, I., \& Finkelstein, E. A. (2020). Anxiety and perceived risk during COVID-19 outbreak. medRxiv.' https://doi.org/10.1101/2020.07.24.20161315

Masten, A. S., \& Narayan, A. J. (2012). Child development in the context of disaster, war, and terrorism: Pathways of risk and resilience. Annual Review of Psychology, 63, 227-257.

Mazza, C., Ricci, E., Biondi, S., Colasanti, M., Ferracuti, S., Napoli, C., \& Roma, P. (2020). A nationwide survey of psychological distress among Italian people during the COVID-19 pandemic: Immediate psychological responses and associated factors. International Journal of Environmental Research and Public Health, 17(9), 3165. https://doi.org/10.3390/ijerph17093165

Meins, E., Centifanti, L. C. M., Fernyhough, C., \& Fishburn, S. (2013). Maternal mind- mindedness and children's behavioral difficulties: Mitigating the impact of low socioeconomic status. Journal of Abnormal Child Psychology, 41(4), 543-553.

Meins, E., \& Fernyhough, C. (2010). Mind-mindedness coding manual, version 2.0 Unpublished manuscript. Durham, UK: Durham University.

Meins, E., Fernyhough, C., Fradley, E., \& Tuckey, M. (2001). Rethinking maternal sensitivity: Mothers' comments on infants' mental processes predict security of attachment at 12 months. The Journal of Child Psychology and Psychiatry and Allied Disciplines, 42(5), 637-648.

Orgilés, M., Morales, A., Delvecchio, E., Mazzeschi, C., \& Espada, J. P. (2020). Immediate psychological effects of the COVID-19 quarantine in youth from Italy and Spain.

Romero, E., López-Romero, L., Domínguez-Álvarez, B., Villar, P., \& Gómez-Fraguela, J. A. (2020). Testing the effects of COVID-19 confinement in Spanish children: The role of parents' distress, emotional problems and specific parenting. International Journal of Environmental Research and Public Health, 17(19), 6975.

Ross, A. M., DeVoe, E. R., Steketee, G., Emmert-Aronson, B. O., Brown, T., \& Muroff, J. (2020). Outcomes of a reflective parenting program among military spouses: The moderating role of social support. Journal of Family Psychology, 34(4), 402-413. https:// doi.org/10.1037/fam0000637

Rutherford, H. J., Wallace, N. S., Laurent, H. K., \& Mayes, L. C. (2015). Emotion regulation in parenthood. Developmental Review, $36,1-14$.

Saddik, B., Hussein, A., Albanna, A., Elbarazi, I., Al-Shujairi, A., Sharif-Askari, F. S., ... \& Halwani, R. (2020). Assessing the influence of parental anxiety on childhood anxiety during the COVID-19 pandemic in the United Arab Emirates. medRxiv.]

Schiborr, J., Lotzin, A., Romer, G., Schulte-Markwort, M., \& Ramsauer, B. (2013). Child-focused maternal mentalization: A systematic review of measurement tools from birth to three. Measurement, 46(8), 2492-2509.

Shai, D., Dollberg, D., \& Szepsenwol, O. (2017). The importance of parental verbal and embodied mentalizing in shaping parental experiences of stress and coparenting. Infant Behavior and Development, 49, 87-96.

Sharp, C., \& Fonagy, P. (2008). The parent's capacity to treat the child as a psychological agent: Constructs, measures and implications for developmental psychopathology. Social Development, 17 , $737-754$

Shigemura, J., Ursano, R. J., Morganstein, J. C., Kurosawa, M., \& Benedek, D. M. (2020). Public responses to the novel 2019 coronavirus (2019-nCoV) in Japan: Mental health consequences and 
target populations. Psychiatry and Clinical Neurosciences, 74(4), 281.

Slade, A. (2005). Parental reflective functioning: An introduction. Attachment \& Human Development, 7, 269-281.

Smaling, H. J., Huijbregts, S. C., Suurland, J., van der Heijden, K. B., Mesman, J., van Goozen, S. H., \& Swaab, H. (2016). Prenatal reflective functioning and accumulated risk as predictors of maternal interactive behavior during free play, the still-face paradigm, and two teaching tasks. Infancy, 21, 766-784.

Spielberger, C. D. (Ed.). (2013). Anxiety: Current trends in theory and research. Elsevier.'

Spinelli, M., Lionetti, F., Pastore, M., \& Fasolo, M. (2020). Parents and Children Facing the COVID-19 Outbreak in Italy. Available at SSRN 3582790.

Suchman, N. E., DeCoste, C., Castiglioni, N., McMahon, T. J., Rounsaville, B., \& Mayes, L. (2010). The Mothers and Toddlers Program, an attachment-based parenting intervention for substance using women: Post-treatment results from a randomizedclinical pilot. Attachment \& Human Development, 12(5), 483-504.
Torales, J., O’Higgins, M., Castaldelli-Maia, J. M., \& Ventriglio, A. (2020). The outbreak of COVID-19 coronavirus and its impact on global mental health. International Journal of Social Psychiatry, 0020764020915212

Whittle, S., Bray, K., Lin, S., \& Schwartz, O. (2020). Parenting and child and adolescent mental health during the COVID-19 pandemic.' https://doi.org/10.31234/osf.io/ag2r7

Zeegers, M. A. J., Colonnesi, C., Stams, G.-J.J.M., \& Meins, E. (2017). Mind matters: A meta-analysis on parental mentalization and sensitivity as predictors of infant-parent attachment. Psychological Bulletin, 143(12), 1245-1272.

Publisher's Note Springer Nature remains neutral with regard to jurisdictional claims in published maps and institutional affiliations. 